\title{
MICROWAVE PROPAGATION IN TOOTH AND DENTAL DEFECT
}

\author{
Maria Papezova1, Dagmar Faktorova ${ }^{2}$
}

\begin{abstract}
INTRODUCTION: The most common method of conventional dental diagnosis involves X-rays, such as Radio Tomography (RT) or Computer Tomography (CT). Such methods are used for diagnosing pores in dental material that can lead to premature failure of dental material. Diagnosis by X-ray provides an objective analysis. However, repeated radiation from X-rays can cause biological damage to human tissues. From this point of view, there is a significant need to progress to quantitative non-invasive and non-destructive testing (NDT) methods to measure dental material and improve treatment options. This article focuses on applying microwave technology to characterize teeth and teeth replacements. Knowledge of microwave propagation in biomaterial with no defects, using a defined microwave frequency range, and subsequently comparing the result with defective material could provide a means of dental diagnosis without the risk of radiation for the patient, i.e. without Xray.
\end{abstract}

OBJECTIVES: The primary objective of this study was to examine microwave technology in the field of dental medical diagnosis as a new NDT method.

METHODS: The basic concept of applying microwave technology to characterize teeth in dental diagnosis was examined using a basic algorithm designed in the MATLAB programming language. Tests used dielectric properties of tooth and tooth decay and propagated electromagnetic (EM) waves to show different characteristics of chosen materials.

RESULTS: The analyses of frequency dependent reflection and transmission coefficients of the chosen material, specifically teeth, at frequency range $0 \mathrm{GHz}$ to $30 \mathrm{GHz}$, computed differences between healthy and defective dental material.

CONCLUSION: Thus, this could be used in providing a dental diagnosis without exposing patients to radiation, i.e. without X-ray. The next stage will involve creating a complete model of a jaw with teeth, and designing a sensor for crack detection for comparisons using this basic algorithm.

UDC Classification: 601 DOI: http://dx.doi.org/10.12955/cbup.v4.828

Keywords: Dental diagnoses, microwave frequencies, transmission coefficients, reflection coefficients, tooth.

\section{Introduction}

The non-destructive testing (NDT) using microwave technology for material characterization involves two basic types: resonant and non-resonant methods. Of these, resonant methods provide the more accurate knowledge of the dielectric properties over a limited frequency range or with a predetermined transmission frequency. The general review aspects of electromagnetic (EM) properties of material, being impedance, speed of waves in materials, permittivity, and permeability are detected by nonresonance methods, though the latter is limited by frequency range (Faktorová et. al., 2015). The EM properties of material are derived from the partial reflection impact of incident wave at an interface between two materials. For this, the tested material has to be placed directly under the wave of transmission for the reflected and transmitted energies to be collected (Faktorová \& Isteniková, 2011). Coaxial, metallic waveguide, dielectric waveguide, planar transmission line, or free space can serve as the transmission line. The EM waves propagate in two ways, first by a free-space method that operates in the EM far-field using antennas, and second by the waveguide methods that operate in the EM near-field using coaxial cables, micro-strip lines, and cavity resonators (Zoughi, 2012). An analysis of the behavior and interaction of EM waves in any human tissue is useful for determining tissue characteristics for medical purposes.

\section{Signal Analysis Technique}

Our aim is to analyze and test biomaterials using microwave frequencies. With these, it is usual to include the sample in a transmission line assembly, organized so as to analyze one or more of it is

\footnotetext{
${ }^{1}$ Maria Papezova, Department of Measurement and Applied Engineering, Faculty of Electrical Engineering, University of Zilina, Zilina, Slovak Republic, maria.papezova@fel.uniza.sk

${ }^{2}$ Dagmar Faktorova, Department of Measurement and Applied Engineering, Faculty of Electrical Engineering, University of Zilina, Zilina, Slovak Republic, dagmar.faktorova@fel.uniza.sk
} 
scattering parameters ( $\boldsymbol{S}$-parameters), which are a function of the dielectric properties (Faktorová \& Isteniková, 2011). The $S$-parameters express changes in EM energy propagating through a multiport transmission line. When the EM wave meets impedance, the line's characteristics change. These $S$ parameters express the response to reflected $\left(\boldsymbol{S}_{11}, \boldsymbol{S}_{22}\right)$ and transmitted $\left(\boldsymbol{S}_{12}, \boldsymbol{S}_{21}\right)$ EM signals at each port. Due to the absorptions of EM energy in normal transmission, a variety of peaks may appear in the $S$-parameters. This type of analysis might show that frequencies (measured in gigahertz, GHz) can detect the presence and composition of certain material (Papezova \& Faktorova, 2016).

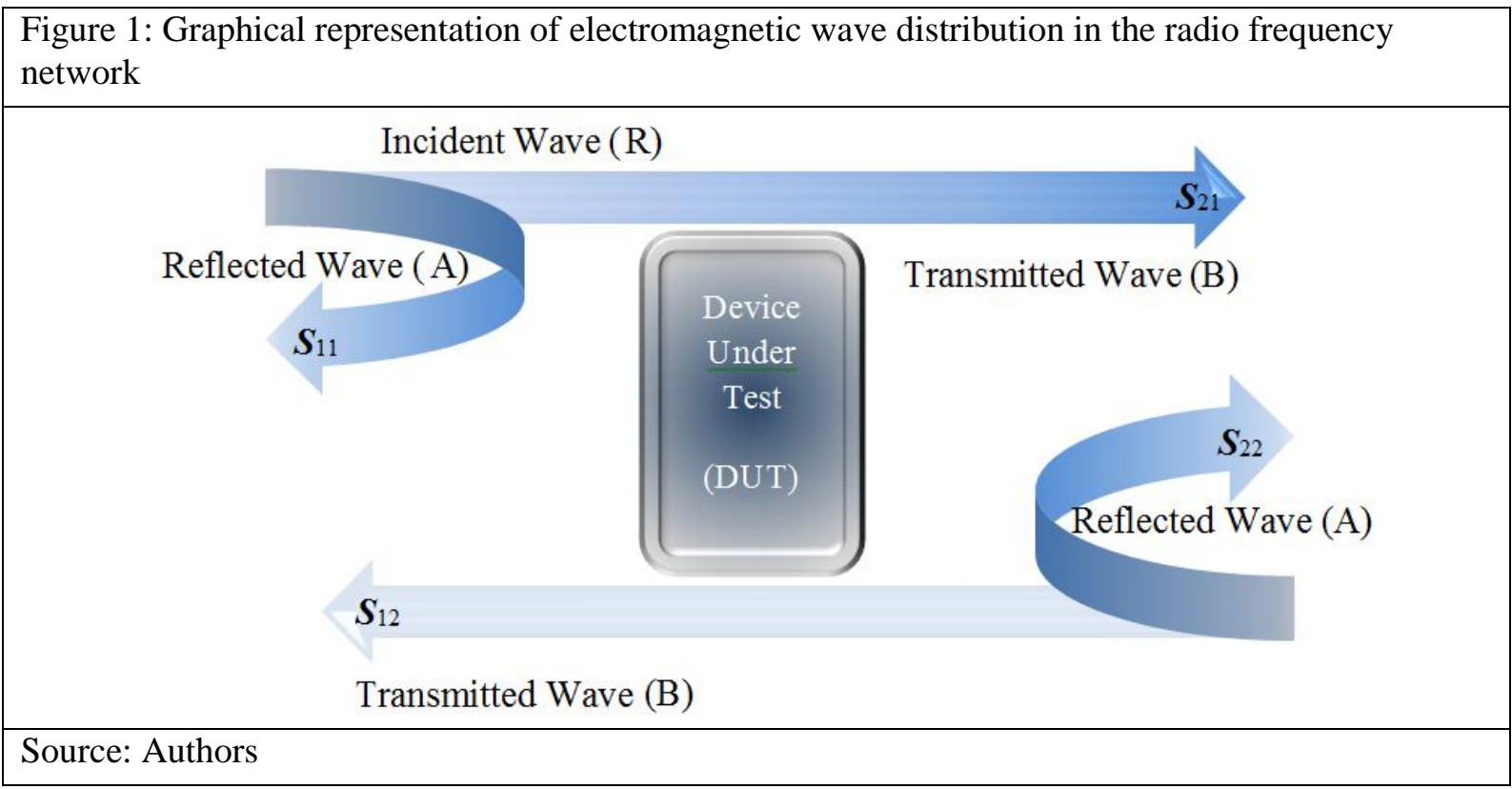

The transmission coefficient, $\mathrm{T}$, is the vector ratio of transmitted wave (B) to the incident wave (R). The reflection coefficient, $\Gamma$, a ratio of reflected wave (A) to the incident wave (R), can be defined as a vector quantity with both magnitude and phase as the ratio of wave. The following Equations 1 and 2 express the coefficients that are shown in Figure 1:

$$
\begin{aligned}
& \mathrm{T}=\frac{\mathrm{B}}{\mathrm{R}} \\
& \Gamma=\frac{\mathrm{A}}{\mathrm{R}}
\end{aligned}
$$

\section{Defining the patterns for examining tooth tissue}

Dielectric properties have values depending on the frequency range. For this purpose, we selected the microwave frequency range $0 \mathrm{GHz}$ to $30 \mathrm{GHz}$. The anatomical structure of teeth comprises precisely defined dimensions of individual parts (Sakaguchi \& Powers, 2012). Each anatomical part and their associated dielectric properties at microwave frequency $(0-30 \mathrm{GHz})$ are shown in Table 1.

From this table is clear that the technology analyzes a tooth as a two-layered structure, because dielectric properties of dentine and enamel are detectable. The technology also detected the structure of gingiva and root. The basic dimension was defined in the algorithm as $6 \times 10^{-3} \mathrm{~m}$ layer of crown and $15 \times 10^{-3} \mathrm{~m}$ layer for cervix and root.

It was necessary to define the complex permittivity of every part of the layer in the algorithm. After relevant formatting of the expression, we calculated the complex relative permittivity with Equation 3 :

$$
\dot{\varepsilon}=\varepsilon_{\mathrm{r}}-j \frac{\delta}{\omega}\left[\mathrm{S} . \mathrm{m}^{-1}\right]
$$


Where:

$\dot{\varepsilon}=$ Complex relative permittivity [-],

$\varepsilon_{\mathrm{r}}=$ Relative permittivity [-],

$\delta=$ Conductivity $\left[\mathrm{S} . \mathrm{m}^{-1}\right]$,

$\omega=$ Angular frequency of harmonic EM wave $\left[\mathrm{s}^{-1}\right]$.

Permittivity describes the interaction of a material with an electric field $\boldsymbol{E}$.

Table 1: Dielectric properties of each part of a human tooth at microwave frequency $(0-30 \mathrm{GHz})$

\begin{tabular}{|l|c|c|c|}
\hline \multicolumn{1}{|c|}{ Tissue } & $\begin{array}{c}\text { Conductivity } \\
{[\delta]}\end{array}$ & $\begin{array}{c}\text { Permittivity } \\
{\left[\varepsilon_{\mathrm{r}}\right]}\end{array}$ & $\begin{array}{c}\text { Loss tangent } \\
{[\operatorname{tg} \delta]}\end{array}$ \\
\hline Dentine (crown) & $1.0 \times 10^{-3}$ & $8.1-8.6$ & 0.14 \\
\hline Enamel (crown) & $1.0 \times 10^{-5}$ & $7.6-8.5$ & 0.07 \\
\hline Gingiva (cervix) & $1.0 \times 10^{0}$ & 12.0 & 0.52 \\
\hline Root (bone) & $9.5 \times 10^{-1}$ & 12.0 & 0.52 \\
\hline
\end{tabular}

Source: Hoshi, Nikawa, Kawai, and Ebisu (1998), Faktorová and Isteniková (2011)

\section{Reflection and Transmission Characteristics of the Two-Layered Tooth Structure}

Using the basic relevant parameter, we analyzed the physical EM wave propagation through the structure described above. In this study, we were focused on analyzing the magnitude of the reflection coefficient, $S_{11}$, and the transmission coefficient, $S_{21}$.

Spectral analysis was chosen for interpreting the results of the EM wave propagation through biological structure. This gave a better understanding of the spread of EM waves through the object by using a spectrogram with "color bar" to show the penetration of depth (Babusiak, Borik, \& Gala, 2014). The resultant scattering parameters of a healthy two-layered human tooth structure are shown at Figure 2.

Figure 2 shows the reflection coefficient of the chosen structure, which oscillated in the frequency range $8 \mathrm{GHz}$ to $22 \mathrm{GHz}$. The oscillation was due to the two-layered nature of the tooth structure. The legend for the depth of penetration in the color map, on the right in Figure 2a and 2b, depicts red as the greatest penetration of EM waves in the object. The greatest penetration of EM waves is from $25 \mathrm{GHz}$ to $30 \mathrm{GHz}$ in a healthy tooth.

Figure 2: $\boldsymbol{S}$-parameters of two-layered tooth structure: a) $\boldsymbol{S}_{11}$ parameter, b) $\boldsymbol{S}_{21}$ parameter
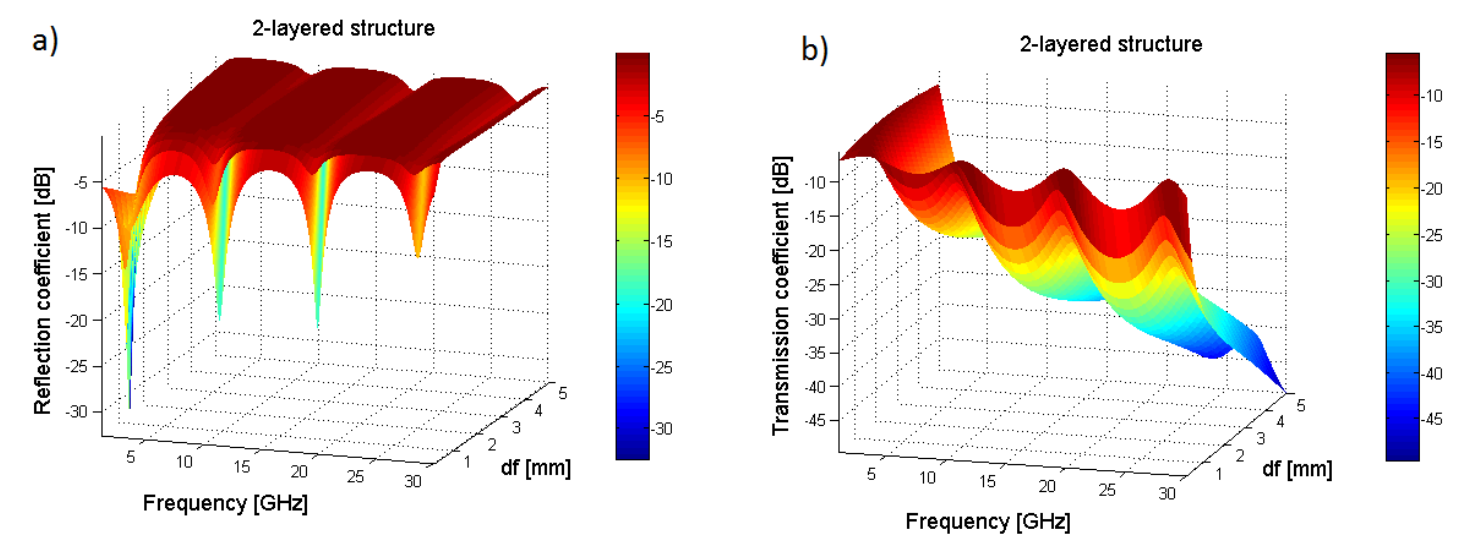

Source: Authors

The transmission coefficient (Figure 2b) refers to a significant decrease in tare of the structure. This decrease is indicated by marked attenuation of EM waves. The maximum depth of penetration is seen at the lowest frequencies, i.e. $2.5 \mathrm{GHz}$. 


\section{Reflection and Transmission Characteristics in Two-Layered Tooth Structures with Decay}

The analysis of the $\boldsymbol{S}$-parameters in tooth tissue with decay is realizable after the analysis of the $\boldsymbol{S}$ parameter in healthy tooth tissue (as described above). The algorithm was modified for decay with tooth decay defined as $1 \times 10^{-3}$ thickness, with complex relative permittivity of $6.04-2.64 \mathrm{j}$, ( $\mathrm{j}$ is the imaginary part of complex permittivity, which is related to the loss of energy within detected object), (Hoshi et al., 1998). Differences between the reflection coefficient in healthy tooth tissue and that of tooth tissue with decay were evident. The reflected waves were mostly absorbed in tooth decay at 2.8 GHz. On the other hand, the greatest penetration of EM waves into the decay occurred at $6.7 \mathrm{GHz}$, as shown by the transmission coefficient (Figure $3 b$ ).

Figure 3: $\boldsymbol{S}$ - parameters of two-layered tooth structure with decay: a) $\boldsymbol{S}_{11}$ parameter, b) $\boldsymbol{S}_{21}$ parameter
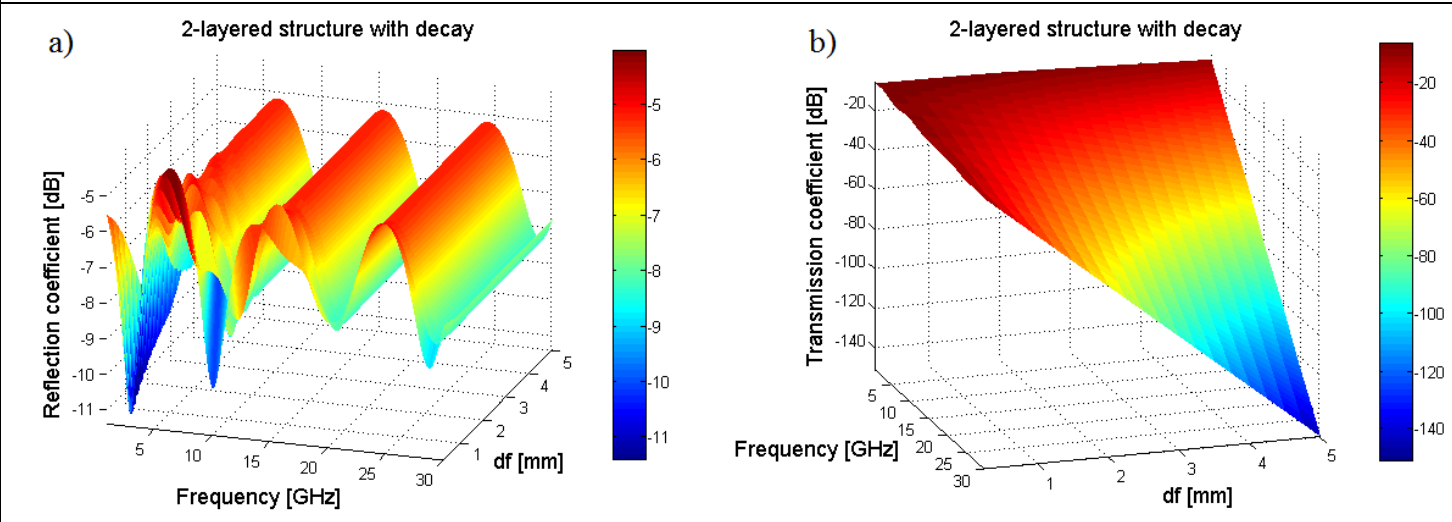

Source: Authors

\section{Conclusion}

The EM properties of material can be derived from the partial reflection impact of the incident wave at the interface between layers using the reflection and transmission coefficient of the EM wave incident on the heterogeneous structure. The coefficients can give information about the quality of contact between two materials with different dielectric properties. The occurrence of some changes between both materials causes change in the reflection coefficient of electromagnetic wave.

From this research, it is possible to determine the dielectric properties of each kind of material, which might act as the foundation for biocompatible material, as well as the dielectric properties of biocompatible materials.

\section{References}

Babusiak, B., Borik, S. \& Gala, M. (2014). Bio-amplifier with Programmable Gain and Adjustable Leads for Basic Measurement of Bioelectric Signals. In Information Technologies in Biomedicine, Volume 4 (pp. 249-260). Springer International Publishing.

Faktorová, D., Pápežová, M., Savin, A., Steigmann, R., Nový, F., \& Bokůvka, O. (2015). Microwave Resonant Methods for Bone Replacement Biomaterials Testing. Procedia Engineering, 100, 1686-1695.

Faktorová, D., \& Isteniková, K. (2011). Modelling of scattering parameters in biological tissues. In: Electrical Review, Vol. 87, No. 5, 2011. pp. 34-36.

Hoshi, N., Nikawa, Y., Kawai, K., \& Ebisu, S. (1998). Application of microwaves and millimeter waves for the characterization of teeth for dental diagnosis and treatment. Microwave Theory and Techniques, IEEE Transactions on, 46(6), 834-838. Lahm, K. (2008). Inmate-on-inmate assault: A multilevel examination of prison violence.

Papezova, M., \& Faktorova, D. (2016). Microwave nondestructive testing of dental materials. International Journal of Applied Electromagnetics and Mechanics, (Preprint), 1-7.

Sakaguchi, R. L., \& Powers, J. M. (2012). Craig's restorative dental materials. Elsevier Health Sciences, 5-18.

Zoughi, R. (2012). Microwave non-destructive testing and evaluation principles (Vol. 4). Springer Science \& Business Media, 13-18. 